\title{
Genetic parameters for faecal egg count following mixed, natural, predominantly Ostertagia circumcincta infection and relationships with live weight in young lambs
}

\author{
S. C. Bishop' ${ }^{1}$ K. Bairden², Q. A. McKellar', M. Park ${ }^{2}$ and M. J. Stear ${ }^{2}$ \\ ${ }^{1}$ Roslin Institute (Edinburgh), Roslin, Midlothian EH25 9PS \\ ${ }^{2}$ Glasgow Unizersity Vetcrinary School, Bearsden Road, Glasgow G61 $1 \mathrm{QH}$
}

\begin{abstract}
Faecal egg counts and live weights were measured on approximately 200 predominantly twin-born Scottish Blackface lambs each year for 3 years, from 1 to 6 months of age. Measurements were made at 4-week intervals following anthelmintic treatment. Heritability estimates (with s.e.s) of log transformed faecal egg count at each age were $0.01,0.00,0.12(0.10), 0.14(0.12), 0.15(0.07)$ and $0.22(0.13)$, for ages 1 to 6 months respectively. Therefore, genetic variation exists for acquired but apparently not for innate resistance to infection. Maternal common environmental effects (with s.e.s) were $0.36(0.11), 0.20(0.05), 0.27(0.09), 0.06(0.08), 0.15(0.09)$ and $0.16(0.08)$, for ages 1 to 6 months respectively. Genetic correlations between faecal egg counts in lambs older than 3 months were not significantly less than 1.0, indicating that faecal egg counts at different ages are expressions of the same trait. Phenotypic correlations between faecal egg counts were generally positive but small. Measurement error contributed one-third of the observed variation for individual egg counts. The heritability of mean faecal egg count from 3 to 6 months was 0.33 (s.e. 0.15), indicating that selection decisions can be made more accurately using multiple egg counts per animal. Phenotypic correlations between faecal egg counts and live weight were generally negative but close to zero. However, genetic correlations between faecal egg counts and live weight in lambs older than 3 months were close to $-1 \cdot 0$, indicating that resistance to gastrointestinal parasites may be an important genetic determinant of growth rate in this environment.
\end{abstract}

Keywords: faecal egg counts, genetic parameters, live weight, Ostertagia, sheep.

\section{Introduction}

Profitable sheep farming relies, in part, upon the efficient control of nematode infection. Currently, the major control strategy is the use of anthelmintics, however this strategy is threatened by the evolution of drug resistance in parasite populations (Jackson, 1993). An alternative, or additional, method for helping to control nematode infection is the selective breeding of sheep with enhanced resistance to infection (Stear and Murray, 1994), especially as selective breeding schemes in Australia and New Zealand have been remarkably successful in improving resistance of sheep to infection with Haemonchus contortus, Irichostrongylus colubriformis and to mixed, natural infections (Piper, 1987; Windon, 1990; Baker et al., 1991; Woolaston and Piper, 1996).
In northern Europe, a prevalent and economically important parasite is the abomasal nematode Ostertagia (Teladorsagia) circumcincta, and this parasite dominates the parasitic fauna of grazing sheep. The genetic parameters of resistance to infection with predominantly $O$. circumcincta are not known, but they are required before the feasibility of selective breeding can be assessed. In addition, a knowledge of the sources of variation among sheep is essential for understanding the infection and for developing sustainable strategies for reducing the effects of parasitic disease.

The alms of this paper are: firstly, to partition the variation observed in faecal egg count in lambs following natural, predominantly $O$. circumcincta infection into components attributable to genetic, 
maternal and measurement error effects, and hence to calculate genetic parameters, secondly, to investigate relationships between faecal egg counts at different ages; and, thirdly, to investigate relationships between faecal egg count and live weight.

\section{Material and methods}

\section{Animals and experimental design}

Data were collected from a commercial flock of Scottish Blackface sheep on an upland farm in southwest Strathclyde, Scotland. Whilst grazing, these sheep were exposed to natural, mixed, predominantly $O$. circumcincta infection. A total of 185, 188 and 194 lambs were studied in 1992, 1993 and 1994, respectively. The lambs were sired by a total of 23 rams, were predominantly twin born and each year were born within a 2 -week period. Lambs were kept in two separate fields prior to weaning each year and after weaning, at about 16 weeks of age, all lambs were kept together on one field to minimize variation in exposure to infective larvae.

Each year faecal samples were collected from the rectum when lambs were 4 weeks of age on average, and thereafter at 4-week intervals until the lambs were 24 weeks of age (26 weeks in 1992 and 1993), giving six samples per animal. Faecal egg counts were made from a 3-g sample of faeces using the modified McMaster technique (Gordon and Whitlock, 1939; Bairden, 1991). Each egg count represented 50 eggs per $\mathrm{g}$. These counts will be referred to as EPG1, EPG2 I... EPG6. In 1993 duplicate aliquots from the same faecal sample were counted for EPG4, EPG5 and EPG6, and in 1994 quadruplicate samples were counted for EPG6. The majority of larvae recovered from culture were $O$. circumcincta. Other parasites identified from the faecal samples but not analysed were from the genera Strongyloides, Nematodirus and Eimeria. After collection of each faecal sample, all lambs were treated with a broad spectrum anthelmintic (Albendazole sulphoxide) which was given at the recommended dose rate of $5 \mathrm{mg} / \mathrm{kg}$ body weight, based on the weight of the heaviest lamb at the time of treatment. The efficacy of the anthelmintic treatment was tested with a faecal egg count reduction test and there was no evidence of drug resistance within this flock. Live weight was also measured at each of the first five sampling dates each year, and will be referred to as WT1,...WT5.

\section{Data analysis}

Preliminary analyses of all traits were undertaken using the GENSTAT statistical package (Lawes Agricultural Trust, 1983) to determine significant fixed effects and test the distributional assumptions for the faecal egg count data. All traits were subsequently analysed fitting fixed effects of sex, birth type and the interaction of field and year of birth, with age at sampling fitted as a covariate. All faecal egg counts (FEC) were positively skewed and were transformed by $\log (\mathrm{FEC}+1)$ prior to analyses.

Genetic parameters were estimated by residual maximum likelihood (REML), fitting an animal model and using a derivative free algorithm to solve the equations (DFREML) (Meyer, 1989). For univariate analyses, additive genetic and maternal common environment components of variance were estimated. Additionally, for the traits of EPG4, EPG5 and EPG6 where replicated egg counts were available, individual animal variance components were also estimated. The data structure was not suitable for estimating maternal genetic effects, and the maternal common environment component may include maternal genetic effects.

Phenotypic and genetic correlations between traits were estimated using the bivariate technique outlined by Thompson et al. (1995). Fitting the same models to each trait as for the univariate analyses did not lead to convergence in the cases attempted, possibly because there were up to 11 (co)variance components to be estimated in each bivariate analysis. Therefore, a simplified model was used in which all dam information was deleted from the data and only the additive genetic and individual animal components of variance were fitted, along with the appropriate covariances and also repeated record effects, where necessary.

Standard errors for each parameter of interest were calculated from the second derivative of the log likelihood, evaluated at the parameter estimate. To enable this derivative to be calculated, a log likelihood profile was obtained by fixing the parameter at points at, and on either side of, the REML parameter estimate and then remaximizing the likelihood for all other variance components.

\section{Results}

Mean values and phenotypic standard deviations for faecal egg count, $\log (\mathrm{FEC}+1)$ and live weight at each of the sampling times are shown in Table 1. Also shown are the maximum values for FEC at each time. A previous paper has discussed the distribution of faecal egg counts using the data collected in 1992 and 1993, and all samples taken from weaning (EPG4) onwards conformed to a negative binomial distribution (Stear et al., 1995a).

Heritabilities $\left(h^{2}\right)$, maternal common environmental $\left(c^{2}\right)$ and individual lamb $\left(i^{2}\right)$ effects are shown in 
Table 1 Mean values and phenotypic standard deciations for faecal egg count (EPG) and live areight (WT) measured at months 1 to 6

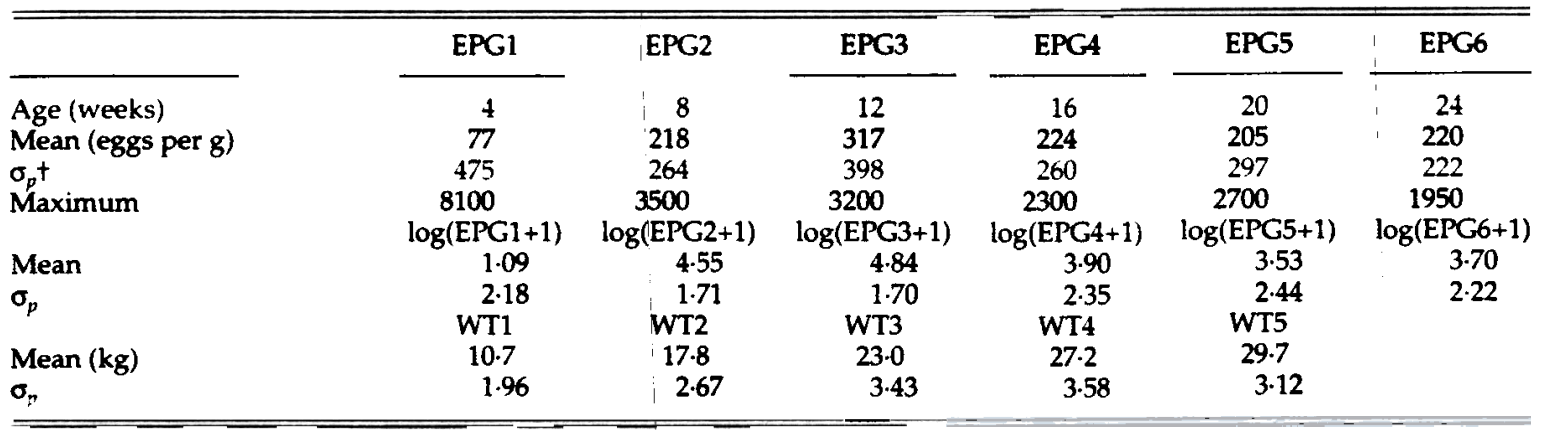

$+\sigma_{p}=$ phenotypic standard deviation

Table 2 Heritabilities, maternai common encironment and individual animal effects for faecal ege count (EPG) measured at months 1 to 6 t

\begin{tabular}{lcccccc}
\hline \hline & EPG1 & EPG2 & EPG3 & EPG4 & EPG5 & EPG6 \\
\hline$h^{2} \ddagger$ & 0.01 & 0.00 & 0.12 & 0.14 & 0.15 & 0.22 \\
s.e. & & & 0.10 & 0.12 & 0.07 & 0.13 \\
$c^{2} \ddagger$ & 0.36 & 0.20 & 0.27 & 0.06 & 0.15 & 0.16 \\
s.e. & 0.11 & 0.05 & 0.09 & 0.08 & 0.09 & 0.08 \\
$i^{2} \ddagger$ & & & & 0.50 & 0.31 & 0.32 \\
\hline \hline
\end{tabular}

+ All data $\log$ transformed prior to analyses.

$\ddagger h^{2}=$ heritability; $c^{2}=$ maternal common. environment effect; $t^{2}=$ individual animal effect.

Table 2. The $t^{2}$ effects were estimated for cases where replicate egg counts were made. The heritabilities of single egg counts were essentially zero at 4 and 8 weeks of age, then increased to a maximum of 0.22 at 24 weeks. In contrast, maternal common environmental $\left(c^{2}\right)$ effects (which may include maternal genetic effects) were large in the very young lambs and decreased as the animal matured, but were still evident 8 weeks after weaning (0.16). The individual lamb $\left(i^{2}\right)$ effects were large, as may be expected, accounting for up to half of the variation at the last three sampling times. The true measurement error $\left(e^{2}\right)$, as defined by the variation between replicated egg counts from the same faecal sample, can be estimated from the variance components in Table 2 for EPG4, EPG5 and EPG6 (i.e. $e^{2}=1-h^{2}-c^{2}$ $-i^{2}$ ). The $e^{2}$ values at these three sampling times were $0.30,0.39$ and 0.30 , respectively. In other words, approximately one-third of the variation observed among animals in faecal egg counts was due solely to measurement error.

An alternative method for estimating $h^{2}$ values for EPG4, EPG5 and EPG6, where there are replicated measurements, is to estimate heritabilities choosing just one measurement per animal per sampling time, repeating this across all possible replicate combinations. Doing this and averaging the values obtained resilted in $h^{2}$ and $c^{2}$ values of 0.14 and $0.0 \mathrm{~h}$ for EPG4, 0.16 and 0.13 for EPG5 and 0.21 and 0.14 for EPG6. These values are close to those shown in Table 2.

Simultaneous analysis of the data from EPG3 to EPG6 gave a heritability estimate for a single egg count of 0.12 (s.e. 0.06), a maternal common environmental effect of 0.06 , an individual animal effect common to all measurements ( $i^{2}$ permanent) of 0.01 , and an individual animal effect specific to each measurement ( $i^{2}$ temporary) of 0.49 . The measurement error effect was 0.31 . The relatively low heritability and maternal effects suggest that the true genetic correlation among egg counts may be less than one and the nature of the maternal influence may differ between sample dates.

Genetic and phenotypic correlations between faecal egg counts at different times are shown in Table 3. No attempt was made to calculate genetic correlations with EPG1 and EPG2 because these traits expressed essentially no genetic variation. Residual correlations, obtained from univariate

Table 3 Phenotypic (above diagonal) and genetic (below diagonal) correlations between faecal egg count (EPG) at months 1 to $6+t$

\begin{tabular}{lrrrr}
\hline & EPG3 & EPG4 & EPG5 & \multicolumn{1}{c}{ EPG6 } \\
\hline EPG1 & \multicolumn{1}{c}{0.09} & 0.01 & 0.02 & 0.06 \\
EPG2 & -0.05 & -0.07 & -0.07 & -0.04 \\
EPG3 & & 0.16 & 0.14 & 0.22 \\
EPG4 & 1.00 & & 0.24 & 0.25 \\
EPG5 & 0.69 & 0.92 & & 0.22 \\
s.e. & 0.26 & 0.25 & & \\
EPG6 & 0.99 & 0.80 & 0.82 & \\
s.e. & 0.23 & 0.33 & 0.25 & \\
\hline
\end{tabular}

t All data $\log$ transformed prior to analyses.

$\ddagger$ Phenotypic correlation (EPG1, EPG2) $=-0.01$ 
GENSTAT REML, rather than phenotypic correlations in the true sense, are presented for these two traits, although for all traits residual and phenotypic correlations were almost identical. All genetic correlations between EPG3, EPG4, EPG5 and EPG6 were large, positive and not significantly different from 1.0, indicating that faecal eggs counts on these four occasions may effectively be expressions of the same trait, or closely related traits.

The pattern of phenotypic correlations between successive faecal egg counts differs from that for genetic correlations. From EPG3 onwards, all measurements were positively correlated with each other, although this correlation was moderate, generally in the range 0.15 to 0.25 . Faecal egg counts at 8 weeks were weakly, negatively, correlated with subsequent measurements, and all correlations with EPG1 were close to zero.

Heritabilities and maternal common environmental effects for live weight are shown in Table 4, along with correlations with faecal egg count. Again, no attempt was made to calculate genetic correlations with EPG1 or EPG2, and residual correlations are shown for these two traits. All live weight heritability values were large, with the exception of the 20-week value, although they are compromised by large standard errors which limit their interpretation. The maternal common environment values were also very high, confirming the strong maternal effects often observed in this breed. Interestingly, the genetic but not the phenotypic correlations among live weight and faecal egg count were large and negative. Phenotypic correlations with growth rate immediately prior to, or immediately after, the time of sampling were similar to those presented in Table 4.

\section{Discussion}

The number of eggs counted in the faeces is a complex interaction between the level of parasite

Table 4 Heritabilities and maternal common environment effects for live weight (WT), and phenotypic and genetic correlations with faecal egg countt

\begin{tabular}{llrrrr}
\hline & WT1 & WT2 & WT3 & WT4 & WT5 \\
\hline$h^{2}$ & 0.41 & 0.50 & 0.43 & 0.28 & 0.14 \\
s.e. & 0.28 & 0.30 & 0.16 & 0.25 & 0.14 \\
& 0.49 & 0.43 & 0.47 & 0.46 & 0.38 \\
s.e. & 0.13 & 0.14 & 0.11 & 0.12 & 0.09 \\
$r_{p}$ & 0.09 & -0.00 & -0.13 & -0.01 & -0.06 \\
$r_{g}$ & & & -0.90 & -1.00 & -0.63 \\
s.e. & & & 0.28 & & 0.32 \\
\hline
\end{tabular}



burden in the host and the fecundity of the adult female parasites, both of which may be regulated by density-dependent factors outside the direct control of the host. Therefore, there may not be a linear relationship between faecal egg count and either an animal's immune response or the actual parasite burden. As such, faecal egg counts may be considered to simply be an indicator of resistance/ susceptibility to parasite infection. Actual non-linear relationships between faecal egg counts and true worm burdens are explored elsewhere (M. J. Stear and S. C. Bishop, unpublished results).

At the population level, many factors may lead to variation in faecal egg counts. Variation between lambs over and above that explicable by genetic background and maternal effects will exist at any given time due simply to, for example, chance effects in exposure to larvae, variations in acquired immunity, variations in density-dependent factors, etc. When actual faecal samples are taken there will also be variation due to the faecal sample collected. that is whether or not the sample is representative in terms of the number of eggs present per unit of faecal material. These factors will all contribute to the observed $i^{2}$ effects. Finally, having obtained the faecal sample, there will inevitably be measurement error, leading to the $e^{2}$ effects observed for EPG4, EPG5 and EPG6.

The existence of additive genetic effects has been reported in Australia and New Zealand for egg counts following infection with $H$. contortus (Woolaston et al., 1991; Woolaston and Piper, 1996), T. colubriformis (Windon, 1990) or mixed natural infections (Baker et al., 1991; Cummins et al., 1991; Bisset et al., 1992; Douch et al., 1995, amongst others). Additive genetic effects for faecal egg counts have also been reported in African sheep (Baker et al., 1994). Although the estimated heritabilities for FEC need not be similar for studies which have different parasite species, different environmental conditions and different experimental protocols, the estimate at 6 months of age $(0.22)$ is remarkably similar to most published estimates which usually lie between 0.2 and 0.3 .

In this study there was little or no detectable genetic variation in faecal egg counts in very young animals. This indicates that there is either no innate resistance to infection or that there is no genetic variation in innate resistance, with genetic variation only existing for acquired resistance. The mechanisms of acquired resistance are not known for any parasite, but experimental results suggest that variation in faecal egg counts following infection with $O$. circumcincta in sheep is due to variation in parasite-specific $\operatorname{IgA}$ responses which regulate parasite fecundity, and 
also to variation in the production of globule leucocytes which are associated with decreased worm burdens (Smith et al., 1985; Stear et al., 1995b).

Maternal common environmental effects accounted for a large but variable proportion of the observed variation in faecal egg counts. There are several possible maternal influences. Firstly, maternal antibodies in milk and colostrum might influence egg counts directly or by interfering with the development of effective immunity in the lamb. The consistent negative correlation between egg counts at 2 months of age and subsequent egg counts could be due to maternal antibodies: those lambs with most maternal antibody are best protected at a young age, but develop immunity more slowly or less effectively. Alternatively, chance differences in parasite exposure might lead to higher counts initially but subsequently to better immune responses and lower counts. A second potential maternal influence could be grazing behaviour. Lambs were kept with their mothers until 4 months of age and lambs often grazed together with their dams. Therefore, dams which tended to graze more heavily infected areas would have lambs with higher than average exposures. Learnt grazing behaviour may also account for maternal effects persisting beyond weaning. Additionally, ewe milk production might affect the amount of grass lambs eat pribr to weaning, and hence their exposure to infective larvae.

The genetic correlations presented in this paper indicate that faecal egg counts from 3 to 6 months of age may be expressions of the same trait. The phenotypic correlations between EPG3, EPG4, EPG5 and EPG6 are, however, considerably lower than the equivalent genetic correlations. This may simply reflect the variation inherent in faecal egg counts, especially as these sheep were given an effective anthelmintic at each sampling date. In terms of variance components, the only terms which contribute to a phenotypic correlation in this example are the genetic covariance, the maternal covariance and the permanent $i^{2}$ component. Simultaneous analyses of EPG3 to EPG6 (see Results) suggested that the latter two components may be small, hence the small observed phenotypic correlations.

The large observed measurement error, approximately one-third of the variation, highlights the importance of distinguishing between the heritability of a single egg count and that of mean faecal egg count. Taking the average faecal egg count from several replicated counts from the same sample will reduce the measurement error and thus increase the heritability. For example, taking the mean of two replicated counts will, theoretically, halve the measurement error term and increase the heritability for EPG6 to 0.26 , although the maximum value obtainable by this means will only be 0.31 . A more effective means of increasing the heritability may be to average faecal egg counts across time, as this will reduce both the $e^{2}$ and the temporary $l^{2}$ effects, and is justified by the high estimated genetic correlations between sampling dates. Assuming that faecal egg counts are made on two separate occasions, then the heritability should increase from 0.12 to 0.20 , and for four sampling times to 0.31 . Averaging all faecal egg counts per animal from EPG3 to EPG6, including the replicated counts, gave a heritability of 0.33 (s.e. 0.15 ), compared with an approximate predicted value of 0.32 obtained when $i^{2}$ (temporary) and $e^{2}$ were scaled by the average number of sampling times per animal and the average number of faecal egg counts made per animal, respectively.

The large, negative genetic correlations between faecal egg count and live weight indicate that resistance to gastrointestinal parasites is probably an important determinant of growth rate, in this environment. These genetic correlations are somewhat stronger than those previously reported between faecal egg count and live weight, most of which are close to and not significantly different from zero (e.g. Bisset et al., 1992; Baker et al., 1991; Eady et al., 1994; McEwan et al., 1995; Douch et al., 1995), although those reported by Bisset et al. (1994) for female lambs under natural infection were stronger, being close to -0.5 . As pointed out by Eady et al. (1994), there is considerable inconsistency amongst reports of genetic correlations (both estimated and implied by correlated responses to selection) between faecal egg counts and production traits. It is not clear why the correlations in this paper differ from previously published results but there does appear to be a strong case for monitoring both faecal egg count and live weight in selective breeding schemes in the United Kingdom, especially if the level of parasite exposure varies between years.

The phenotypic correlations between live weight and faecal egg count were low, in agreement with most published results. The same arguments may be used to rationalize these results as were used to account for the low correlations between successive faecal egg counts. In other words, a single faecal egg count is a very variable entity. Moreover, live weight at any given age is a cumulative effect of all the experiences that the lamb has had up to that age, whereas a faecal egg count simply describes the state of being at that point in time, for the current infection. Correlations were also calculated between live weight and the mean faecal egg count up to that point in time. Again, the genetic correlations were 
always close to -1.0 , whereas the phenotypic correlations were negative but small.

In summary, we have demonstrated that genetic variation exists for acquired, but not for innate resistance to infection to $O$. circumcincta infection in Scottish Blackface lambs under Scottish grassland conditions, with maternal common environment effects also contributing significantly to the variation. In this data set, faecal egg counts taken at different ages are, genetically, strongly correlated with each other and genetically negatively correlated with live weight. Therefore, selective breeding, using the average of several faecal egg counts, may offer a desirable and feasible option in assisting in the control of nematode infections.

\section{Acknowledgements}

We thank the Overseas Development Organisation for financial support and Professors J. L. Duncan, P. H. Holmes and $M$. Murray for encouragement and advice. SCB is grateful to the Ministry of Agriculture, Fisheries and Food for financial assistance.

\section{References}

Bairden, K. 1991. Ruminant parasitic gastroenteritis: some observations on epidemiology and control. Ph.D. thesis, University of Glasgow.

Baker, R. L., Mwamachi, D. M., Audho, J. O. and Thorpe, W. 1994. Genetic resistance to gastrointestinal nematode parasites in Red Masai sheep in Kenya. Proceedings of the fifth world congress on genetics applied to livestock production, Guelph, vol. 20, pp. 277-280.

Baker, R. L., Watson, T. G., Bisset, S. A., Vlassof, A. and Douch, P. G. C. 1991. Breeding sheep in New Zealand for resistance to internal parasites: research results and commercial application. In Breeding for disease resistance (ed. G. D. Gray and R. R. Woolaston), pp. 19-32. Australian Wool Corporation, Melbourne.

Bisset, S. A., Morris, C. A., Squire, D. R., Hickey, S. M. and Wheeler, M. 1994. Genetics of resilience to nematode parasites in Romney sheep. New Zealand Journal of Agricultural Research 37: 521-534.

Bisset, S. A., Vlassof, A., Morris, C. A., Southey, B. R. and Baker, R. L. 1992. Heritability of and genetic correlations among faecal egg counts and productivity traits in Romney sheep. New Zealand Journal of Agricultural Research 35: 51-58.

Cummins, L. J., Thompson, R. L., Yong W. K., Riffkin, G. G., Goddard, M. E., Callinan, A. P. L. and Saunders, M. J. 1991. Genetics of Ostertagia selection lines. In Breeding for disease resistance (ed. G. D. Gray and R. R. Woolaston), pp. 11-18. Australian Wool Corporation, Melbourne.

Douch, P. G. C., Green, R. S., Morris, C. A., Bisset, S. A., Vlassof, A., Baker, R. L., Watson, T. G., Hurford, A. P. and Wheeler, M. 1995. Genetic and phenotypic relationships among anti-Trichostrongylus colubriformis antibody level, faecal egg count and body-weights in grazing Romney sheep. Livestock Production Science 41: 121-132.
Eady, S. J., Woolaston, R. R. and Mortimer, S. I. 1994. Internal parasite resistance of merino flocks selected for production. Proceedings of the fifth world congress on genetics applied to livestock production, Guelph, vol. 20, pp. 289-292.

Gordon, H. McL. and Whitlock, H. V. 1939. A new technique for counting nematode eggs in sheep faeces. Journal of the Council for Scientific and Industrial Research, Australia 12: 50-52.

Jackson, F. 1993. Anthelmintic resistance - the state of play. British Veterinary Journal 149: 123-138.

Lawes Agricultural Trust. 1983. GeNSTAT a general statistical program. Numerical Algorithms Group Limited.

McEwan, J. C., Dods, K. G., Bain, W. E., Duncan, S. J., Wheeler, R., Knowler, K. J., Reid, P. J., Green, R. S. and Douch, P. G. C. 1995. Genetic estimates for parasite resistance traits in sheep and their correlations with production traits. New Zealand Journal of Zoology 22: 177.

Meyer, K. 1989. Restricted maximum likelihood to estimate variance components for animal models with several random effects using a derivative-free algorithm. Génétique, Sélection et Evolution 21: 317-340.

Piper, L. R. 1987. Genetic variation in resistance to internal parasites. In Merino improvement programs in Australia (ed. B. J. McGuirk), pp. 351-363. Australian Wool Corporation, Melbourne.

Smith, W. D., Jackson, F., Jackson, E. and Williams, J. 1985. Age immunity to Ostertagia circumcincta: comparison of the local immune responses of $4 \frac{1}{2}$ and 10 month old lambs. Journal of Comparative Pathology 95: 235-245.

Stear, M. J., Bairden, K., Duncan, J. L., Gettinby, G., McKellar, Q. A., Murray, M. and Wallace, D. S. 1995a. The distribution of faecal nematode egg counts in Scottish Blackface lambs following natural, predominantly Ostertagia circumcincta infection. Parasitology 110: 573-581.

Stear, M. J., Bishop, S. C., Doligalska, M., Duncan, J. L., Holmes, P. H., Irvine, J., McCririe, L., McKellar, Q. A., Sinski, F. and Murray, M. 1995b. Regulation of egg production, worm burden, worm length and worm fecundity by host responses in sheep infected with Ostertagia circumcincta. Parasite Immunology 17: 643-652.

Stear, M. J. and Murray, M. 1994. Genetic resistance to parasitic disease: particularly of resistance in ruminants to gastrointestinal nematodes. Veterinary Parasitology 54: 161-176.

Thompson, R., Crump, R. E., Juga, J. and Visscher, P. M. 1995. Estimating variances and covariances for bivariate animal models using scaling and transformation. Genetics, Selection, Evolution 27: 33-42.

Windon, R. G. 1990. Selective breeding for the control of nematodiasis in sheep. Revue Scientific et Technique de l'Office International des Epizooties 9: 555-576.

Woolaston, R. R. and Piper, L. R. 1996. Selection of merino sheep for resistance to Haemonchus contortus: genetic variation. Animal Science 62: 451-460.

Woolaston, R. R., Windon, R. G. and Gray, G. D. 1991. Genetic variation in resistance to internal parasites in Armidale experimental flocks. In Breeding for disease resistance (ed. G. D. Gray and R. R. Woolaston), pp. 1-9. Australian Wool Corporation, Melbourne.

(Received 14 September 1995-Accepted 6 July 1996) 